\title{
EDITORIAL
}

\section{La innovación en cirugía. ¿Cuál es el límite?}

The innovation in surgery: which is the limit?

DOI: $10.31837 /$ cir.urug/3.1.1

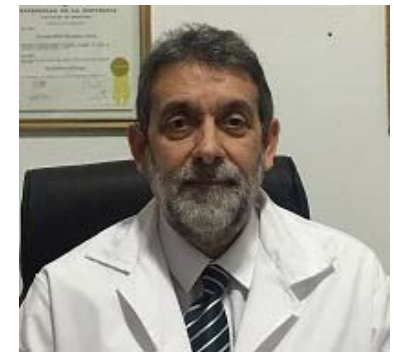

La innovación y el generar hechos nuevos es propio de la condición humana y están presentes desde el comienzo de nuestra especie; desde la invención de la rueda hasta los viajes espaciales, pasando por la antisepsia, la anestesia, desarrollo de antibióticos, nuevas técnicas quirúrgicas, cirugía laparoscópica, robótica, impresión 3D y nuevas tecnologías en las diversas áreas, etc.

El creciente interés por este tema en relación con la Cirugía queda claramente demostrado cuando revisamos las publicaciones luego de una búsqueda en la base de datos PubMed utilizando los términos innovación y cirugía.

En 1986 se publicaron 23 artículos, 65 artículos en 1996, 193 en 2006, 1929 en 2016 y en una reciente búsqueda en noviembre de 2018 encontramos 10.948 artículos.

La Real Academia Española contempla dos acepciones para el término innovar:

1. Mudar o alterar algo introduciendo novedades.

2. Creación o modificación de un producto y su introducción en un mercado.

La innovación en cirugía puede entenderse como un proceso dinámico y continuo que comprende la introducción de una nueva tecnología o técnica, la cual inicia o genera un cambio en la práctica clínica, pero que además supone un riesgo para el paciente y el médico que la aplica.

Para Mayol Martínez innovar es utilizar el conocimiento para generar valor.

El valor se define como el cociente entre la efectividad clínica (EC) más la calidad percibida (CP) menos los efectos adversos (EA) en relación con los costos (C):

$\mathrm{EC}+\mathrm{CP}-\mathrm{EA} / \mathrm{C}$.

El progreso de la cirugía ha ido de la mano de la innovación, con lo que se ha logrado una cirugía más segura, disminuir las complicaciones y la mortalidad, así como mejorar la calidad de vida de los pacientes.

Esta innovación durante muchísimos años fue constante y esporádica, permitiendo su análisis y evaluación de los resultados para definir luego si se incorporaba a la práctica 
clínica o era dejada de lado; así como cuál era el mejor modo de adquirir las competencias necesarias para poder realizarlas en forma segura.

Pellegrini la compara a la navegación por un río con aguas tranquilas intercalados con momentos de corrientes más rápidas que luego vuelven a la calma y permiten reacomodarse, evaluar la situación y continuar el viaje.

En los últimos años ha habido un cambio en la velocidad en la cual ocurren los cambios tecnológicos producto de la innovación. Actualmente es posible compararlo a la navegación por un río torrentoso y con aguas turbulentas, con la aparición frecuente de nuevas tecnologías que obliga a que los plazos sean más breves para evaluar y definir su incorporación o no.

De acuerdo con Gray la innovación abarca tres áreas: técnicas, organización de la práctica y nuevos instrumentos y dispositivos innovadores.

Las innovaciones se pueden agrupar en diferentes grupos:

1. Innovación básica, fundamental o radical es la que inicia una nueva disciplina y en general se puede definir en forma precisa cronológica y biográficamente. Son ejemplos de ella la aplicación de los rayos X a la práctica clínica (Roentgen), Anestesia, anastomosis vasculares (Alexis Carrel), trasplante cardíaco (C. Barnard), bypass aortocoronario (R. Favaloro), robótica e impresión 3D entre otras.

2. Innovación incremental, permite mejorar de manera significativa algo ya inventado, sin modificar sus características fundamentales: cambios moleculares de algún fármaco, el cambio del endoscopio rígido por el flexible, la introducción de la laparoscopía y toracoscopía como métodos menos invasivos sin cambiar básicamente los procedimientos quirúrgicos ya validados.

3. Otro grupo corresponde a la innovación menor que no representa un cambio o innovación importante pero que ayuda a resolver problemas clínicos con muy bajo riesgo para el paciente, como por ejemplo una modificación técnica menor a un procedimiento ya aceptado.

No toda innovación es necesaria ni buena para nuestros pacientes y muchas de ellas quedan por el camino y son desechadas. Pero otras pueden significar un avance y una oportunidad de mejorar diagnósticos y /o tratamientos, por lo que deben ser sometidas a evaluación en forma rápida.

El no posicionarnos en el lugar adecuado en estos tiempos nos expone a no utilizar la mejor opción de diagnóstico y tratamiento a nuestros pacientes y en segundo lugar, 
dejar la puerta abierta al ingreso de la competencia internacional que ya empieza a instalarse en nuestro país.

Los cirujanos no podemos quedar por fuera del progreso tecnológico; tenemos un imperativo ético que nos obliga a ser parte activa en estos procesos.

Fried (ex presidente del Colegio Americano de Cirujanos) propone plantearse cuatro interrogantes frente a la aparición de un nuevo dispositivo o una nueva tecnología:

1. ¿ ¿Responde a una necesidad clínica, ofrece una solución a un problema clínico real?

2. ¿Les agrega valor a las opciones ya existentes?

3. ¿Es económicamente viable?

4. ¿ ¿Puede ser realizada por la mayoría de los cirujanos o solo por una elite?

Los cirujanos tenemos como desafíos permanentes el análisis crítico acerca de:

a. Qué criterios de evaluación y validación deben aplicarse durante la introducción de nuevas tecnologías o procedimientos. La eficacia y seguridad del procedimiento requieren ser validadas mediante aplicación de la metodología científica (ensayos clínicos aleatorizados, meta-análisis)

b. Cuáles deben ser los requerimientos de entrenamiento de los

cirujanos para incorporar una nueva tecnología, cuál debe ser la curva de aprendizaje necesaria para maximizar la seguridad del paciente. Cabe destacar el rol importante que tiene la simulación y su impacto en la adquisición de competencias y destrezas del cirujano.

c. Cómo deben evaluarse los cirujanos para determinar si están aptos para desarrollar un nuevo procedimiento

Los principios bioéticos que rigen el accionar médico deben ser preservados: no maleficencia (primun non nocere), beneficencia, información veraz al paciente así como su consentimiento acerca del procedimiento, riesgos, complicaciones, resultados esperables y experiencia del equipo en el tratamiento a realizar.

Una mención especial (que excede el objetivo de este editorial) merecen los conflictos de compromisos y los conflictos de interés que pueden sesgar la toma de decisiones.

Coincido con Burdiles en cuanto a que la intención de transparentar estos conflictos a través de los "disclosures" impuestos por los anglosajones como solución o atenuante no es suficiente para generar confianzas más profundas y duraderas entre la sociedad y el cuerpo médico. 
La innovación es el motor en el progreso de la cirugía. Es imprescindible el análisis y la evaluación crítica de esta innovación en forma permanente.

Es necesaria su regulación a través de los colegios profesionales, sociedades científicas, Facultades de Medicina, autoridades sanitarias y pacientes.

Dr. Gustavo Rodríguez Temesio

Editor Jefe

\section{Bibliografía}

1. Rogers WA, Lotz M, Hutchison K, Pourmoslemi A, Eyers A. Identifying surgical innovation: a qualitative study of surgeons’ views. Ann Surg. 2014; 259:273-8.

2. Mayol Martínez J. Innovacion en cirugía. Cir. Esp 2016;94:207-9.

3. Porter ME. What is value in health care? N Engl J Med. 2010;363:2477-81

4. Burdiles P., Ortiz A, Castro M. Innovación en Medicina: una mirada desde la bioética Rev. Med. Clin. Condes 2012;23:492-501

5. Gray M, El Turabi A. Optimizing the value of interventions for populations BMJ 2012;345:e6192

6. Dewar RD, Dutton JE. The adoption of radical and incremental innovations: an empirical analysis. Management science 1986; 32:1422-1433.

7. Fried GM. Presidential Address. The challenges of change: Presidential Address to the 69th Annual Meeting of the Central Surgical Association Madison, Wisconsin, March 2012. Surgery 2012;152:509-16. 